TRANSACTIONS OF THE

AMERICAN MATHEMATICAL SOCIETY

Volume 350, Number 11, November 1998, Pages 4499-4519

S 0002-9947(98)01952-7

\title{
TRACE ON THE BOUNDARY FOR SOLUTIONS OF NONLINEAR DIFFERENTIAL EQUATIONS
}

\author{
E. B. DYNKIN AND S. E. KUZNETSOV
}

ABstract. Let $L$ be a second order elliptic differential operator in $\mathbb{R}^{d}$ with no zero order terms and let $E$ be a bounded domain in $\mathbb{R}^{d}$ with smooth boundary $\partial E$. We say that a function $h$ is $L$-harmonic if $L h=0$ in $E$. Every positive $L$-harmonic function has a unique representation

$$
h(x)=\int_{\partial E} k(x, y) \nu(d y)
$$

where $k$ is the Poisson kernel for $L$ and $\nu$ is a finite measure on $\partial E$. We call $\nu$ the trace of $h$ on $\partial E$.

Our objective is to investigate positive solutions of a nonlinear equation

$$
L u=u^{\alpha} \quad \text { in } E
$$

for $1<\alpha \leq 2$ [the restriction $\alpha \leq 2$ is imposed because our main tool is the $\alpha$-superdiffusion which is not defined for $\alpha>2$ ]. We associate with every solution $u$ a pair $(\Gamma, \nu)$, where $\Gamma$ is a closed subset of $\partial E$ and $\nu$ is a Radon measure on $O=\partial E \backslash \Gamma$. We call $(\Gamma, \nu)$ the trace of $u$ on $\partial E$. $\Gamma$ is empty if and only if $u$ is dominated by an $L$-harmonic function. We call such solutions moderate. A moderate solution is determined uniquely by its trace. In general, many solutions can have the same trace. We establish necessary and sufficient conditions for a pair $(\Gamma, \nu)$ to be a trace, and we give a probabilistic formula for the maximal solution with a given trace.

\section{INTRODUCTION}

1.1. Diffusions. We start from an elliptic differential operator

$$
L u=\sum_{i, j} a_{i j} \frac{\partial^{2} u}{\partial x^{i} \partial x^{j}}+\sum_{i} b_{i} \frac{\partial u}{\partial x^{i}}
$$

in $\mathbb{R}^{d}$. An $L$-diffusion in $\mathbb{R}^{d}$ is a Markov process $\xi=\left(\xi_{t}, \Pi_{x}\right)$ with continuous paths and with the transition function $p_{t}(x, y) d y$, where $p_{t}(x, y)$ satisfies the following conditions:

1.1.A. For all $t, x$,

$$
\int_{E} p_{t}(x, y) d y=1
$$

Received by the editors March 28, 1996 and, in revised form, October 16, 1996.

1991 Mathematics Subject Classification. Primary 60J60, 35J60; Secondary 60J80, 60J45, 35J65.

Partially supported by National Science Foundation Grant DMS-9301315.

(C) 1998 American Mathematical Society 
1.1.B. For all $s, t>0$ and all $x, z$,

$$
\int_{\mathbb{R}^{d}} p_{s}(x, y) d y p_{t}(y, z)=p_{s+t}(x, z) .
$$

1.1.C. For every bounded positive continuous function $\varphi$,

$$
v_{t}(x)=\int_{E} p_{t}(x, y) \varphi(y) d y
$$

is the minimal solution of the boundary value problem

$$
\begin{array}{cc}
\frac{\partial v}{\partial t}=L v \quad \text { for } t>0, \\
v \rightarrow \varphi \quad \text { as } t \downarrow 0 .
\end{array}
$$

The existence of such process is proved, under broad conditions on the coefficients of $L$, for instance, in [1].

An $L$-diffusion in an arbitrary open set $D$ is obtained by killing $\xi$ at the first exit time $\tau$ from $D$. Its transition density is given by the formula ${ }^{1}$

$$
p_{D, t}(x, y)=p_{t}(x, y)-\Pi_{x}\left\{\tau<t, p_{t-\tau}\left(\xi_{\tau}, y\right)\right\} .
$$

The corresponding Green's function

$$
g_{D}(x, y)=\int_{0}^{\infty} p_{D, t}(x, y) d t
$$

is finite for all $x \neq y$ if $D$ is bounded. The Green's operator acts on positive Borel functions by the formula

$$
G_{D} \varphi(x)=\int_{E} g_{D}(x, y) \varphi(y) m(d y) .
$$

We fix a bounded domain $E$ with boundary $\partial E$ of class $C^{2, \lambda}$, and we drop the subscripts $E$ in notation $p_{E}, g_{E}, \ldots$. We denote the first exit time from $E$ by $\zeta$.

The name $L$-harmonic functions is used for solutions of the equation $L h=0$ in $E$. Every positive $L$-harmonic function $h$ has a unique representation

$$
h(x)=\int_{\partial E} k(x, y) \nu(d y),
$$

where $k$ is the Poisson kernel for $L$ (equal to the normal derivative of $g(x, y)$ with respect to $y$ ) and $\nu$ is a finite measure on $\partial E$. We call $\nu$ the trace of $h$ on $\partial E$.

The following bounds for $k$ can be found in [19, Lemma 1]:

$$
c|x-y|^{-d} \leq \frac{k(x, y)}{d(x, \partial E)} \leq c^{-1}|x-y|^{-d} \quad \text { for all } x \in E, y \in \partial E
$$

(the constant $c>0$ depends only on $L$ and $E$ ). Formula (1.4) implies that

$$
h(x) \rightarrow 0 \quad \text { as } x \rightarrow a, x \in E,
$$

if $\nu$ does not charge a neighborhood of $a$.

Let $\sigma$ be the measure on $\partial E$ which appears in the representation (1.3) of the $L$-harmonic function $h=1$. Then for all $x$ and $\Gamma$,

$$
\Pi_{x}\left\{\xi_{\zeta} \in \Gamma\right\}=\int_{\Gamma} k(x, y) \sigma(d y) .
$$

\footnotetext{
${ }^{1}$ Writing $\Pi\left\{\Omega^{\prime}, Y\right\}$ means $\int_{\Omega^{\prime}} Y d \Pi$.
} 
1.2. Superdiffusions. A superdiffusion is a mathematical model of a random cloud. The spatial motion of its infinitesimal parts is described by the $L$-diffusion $\xi=\left(\xi_{t}, \Pi_{x}\right)$ in $E$, and the branching mechanism is determined by a parameter $\alpha \in(1,2]$. To every open subset $D$ of $E$ and to every $\mu \in \mathcal{M}(E)^{2}$ there corresponds a random measure $\left(X_{D}, P_{\mu}\right)$ on $E \backslash D$, called the exit measure from $D$. $X_{D}$ describes the mass distribution of the cloud instantaneously frozen on $E \backslash D$, and $P_{\mu}$ is a probability measure corresponding to initial mass distribution $\mu$. All $P_{\mu}$ have the same domain $\mathcal{F}$. For every positive Borel function $f$,

$$
P_{\mu} \exp \left\langle-f, X_{D}\right\rangle=\exp \langle-u, \mu\rangle,
$$

where

$$
u+\mathcal{E}_{D}(u)=K_{D} f
$$

with

$$
\begin{gathered}
\mathcal{E}_{D}(u)(x)=G_{D}\left(u^{\alpha}\right)(x), \\
K_{D} f(x)=\Pi_{x} f\left(\xi_{\tau}\right)
\end{gathered}
$$

( $\tau$ is the first exit time of $\xi$ from $D$ ).

We denote by $P_{x}$ the measure $P_{\delta_{x}}$ corresponding to Dirac's measure at point $x$.

The joint probability distribution of $X_{D_{1}}, \ldots, X_{D_{n}}$ is determined by (1.7) and by the Markov property: for every positive $\mathcal{F}_{\supset D}$-measurable $Y$,

$$
P_{\mu}\left\{Y \mid \mathcal{F}_{\subset D}\right\}=P_{X_{D}} Y,
$$

where $\mathcal{F}_{\subset D}$ is the $\sigma$-algebra generated by $X_{D^{\prime}}$ with $D^{\prime} \subset D$ and $\mathcal{F}_{\supset D}$ is the $\sigma$ algebra generated by $X_{D^{\prime \prime}}$ with $D^{\prime \prime} \supset D$.

The existence of a family $\left(X_{D}, P_{\mu}\right)$ subject to conditions (1.7) and (1.11) is proved in [2].

It follows from (1.7)-(1.10) that

$$
P_{\mu}\left\langle f, X_{D}\right\rangle=\left\langle K_{D} f, \mu\right\rangle .
$$

1.3. Markov process $\left(X_{t}, P_{\mu}\right)$. Besides exit measures $X_{D}$ we also consider random measures $X_{t}$ which describe the mass distribution at a fixed time $t$. For every positive Borel function $f$,

$$
P_{\mu} \exp \left\langle-f, X_{t}\right\rangle=\exp \left\langle-v_{t}, \mu\right\rangle,
$$

where

$$
v_{t}(x)+\Pi_{x} \int_{0}^{t} v_{t-s}\left(\xi_{s}\right)^{\alpha} d s=\Pi_{x} f\left(\xi_{t}\right)
$$

with Random measures $\left(X_{t}, P_{\mu}\right)$ form a Markov process in the state space $\mathcal{M}$. This is a more traditional model of superdiffusion than the model described in Section 1.2 .

${ }^{2}$ We denote by $\mathcal{M}(S)$ the space of all finite measures on a measurable space $S$. 
1.4. Range and polar sets. Consider the class $\mathbb{C}$ of all closed random sets $C(\omega) \subset E \cup \partial E$ with the property that every exit measure $X_{D}$ is concentrated, a.s. ${ }^{3}$ on $C$. There exists a minimal element of $\mathbb{C}$ and it is defined uniquely up to indistinguishability. We denote it by $\mathcal{R}$ and call it the range of $X$. A set $B \subset \partial E$ is called $\mathcal{R}$-polar if

$$
P_{x}\{\mathcal{R} \cap B=\emptyset\}=1 \quad \text { for all } x \in D .
$$

A Borel set $B$ is $\mathcal{R}$-polar if and only if all compact subsets of $B$ are $\mathcal{R}$-polar.

1.5. Principal results. Our objective is to study the class $\mathcal{U}$ of all positive solutions of the equation

$$
L u=u^{\alpha} \quad \text { in } E,
$$

where $E$ is a domain of class $C^{2, \lambda}$ in $\mathbb{R}^{d}$ and $1<\alpha \leq 2$. We say that a solution $u$ is moderate if it is dominated by an $L$-harmonic function. Every positive $L$-harmonic function has a unique representation

$$
h(x)=\int_{\partial E} k(x, y) \nu(d y),
$$

where $k$ is the Poisson kernel for $L$ and $\nu$ is a finite measure on $\partial E$.

The following result is proved in [7].

Theorem 1.1. The formula

$$
u+\mathcal{E}(u)=h
$$

establishes a 1-1 correspondence between moderate solutions $u$ and L-harmonic functions $h$ such that the measure $\nu$ in (1.17) does not charge any $\mathcal{R}$-polar set. The function $h$ is the minimal L-harmonic majorant of $u$, and $u$ is the maximal solution dominated by $h$.

We call $\nu$ the trace of the moderate solution $u$. In Section 3 we prove

Theorem 1.2. If $u$ is an arbitrary positive solution of (1.16) then, for every compact subset $B$ of $\partial E$, there exists the maximal solution $u_{B}$ dominated by $u$ and equal to 0 on $\partial E \backslash B .^{4}$ There exist: (a) a maximal open subset $O$ of $\partial E$ such that $u_{B}$ is moderate for every compact $B \subset O$; (b) a measure $\nu$ on $O$ such that, for every compact $B \subset O$, the trace of $u_{B}$ coincides with the restriction of $\nu$ to $B$.

We call $\Gamma=\partial E \backslash O$ the singular set of $u$ and we call the pair $(\Gamma, \nu)$ the trace of $u$.

We say that $x$ is an explosion point of a measure $\nu$ if $\nu(U)=\infty$ for every neighborhood $U$ of $x$.

Theorem 1.3. A pair $(\Gamma, \nu)$ is the trace of a solution if and only if:

(i) $\Gamma$ is compact;

(ii) $\nu$ is the Radon measure on $O=\partial E \backslash \Gamma$;

(iii) $\nu$ does not charge any $\mathcal{R}$-polar set;

(iv) the conditions:

$$
\Lambda \subset \Gamma \text { is } \mathcal{R} \text {-polar and contains no explosion points of } \nu,
$$

imply that $\Lambda=\emptyset$.

\footnotetext{
${ }^{3}$ Writing a.s. means $P_{x}$-a.s. for all $x \in E$.

${ }^{4}$ Writing $u=f$ at $a \in \partial D$ means $u(x) \rightarrow f(a)$ as $x \rightarrow a, x \in D$. We write $u=f$ on $B \subset \partial D$ if $u=f$ at all $a \in B$.
} 
Theorem 1.3 follows from 3.5.A and Theorems 5.3, 4.2 and 5.2.

A measure $\nu$ is called $\Sigma$-finite if there exist finite measures $\nu_{n}$ such that $\nu=$ $\nu_{1}+\cdots+\nu_{n}+\ldots$

Theorem 1.4. To every $\Sigma$-finite measure $\nu$ on $\partial E$ which charges no $\mathcal{R}$-polar set there corresponds a continuous additive functional $A^{\nu}$ of the superdiffusion $X$. If $(\Gamma, \nu)$ satisfies conditions (i)-(iv) in Theorem 1.3, then

$$
u(x)=-\log P_{x}\left\{\mathcal{R} \cap \Gamma=\emptyset, e^{-A_{\infty}^{\nu}}\right\}
$$

is the maximal element of $\mathcal{U}$ with the trace $(\Gamma, \nu)$.

Theorem 1.4 follows from Theorems 4.2, 4.3 and 6.1 .

1.5. Bibliographical notes. The problem stated at the beginning of Section 1.4 has been studied recently by probabilistic and purely analytic methods. The work of analysts influenced the work of probabilists and vice versa.

It was shown in [2, Section 5.5] that an arbitrary positive solution of equation (1.16) has a representation of the form

$$
u(x)=-\log P_{x} e^{-Z}
$$

where $Z$ is a functional of the superdiffusion $X$. Sufficient conditions on $Z$ were established under which $u$ given by (1.21) is a solution of (1.16). This way a 1-1 correspondence was established between all positive solutions of (1.16) and a certain class of functionals $Z$ of $X$. [Analogous results in a parabolic setting are presented in more detail in [4, Section II.5].]

A boundary value problem

$$
L u=u^{\alpha} \quad \text { in } E, \quad u=\nu \quad \text { on } \partial E
$$

with a finite measure $\nu$ was discussed in [5, p.5]. In particular, it was conjectured that (1.22) has a solution if and only if $\nu$ does not charge any $\mathcal{R}$-polar set. The conjecture was proved in [7], where problem (1.22) was interpreted as the integral equation (1.18) with $h$ given by (1.17). ${ }^{5}$ The results of [7] (summarized in Theorem 1.1) describe all moderate solutions of (1.1).

It was proved in $[6$, Theorem $1.2 \mathrm{a}]$ that the class of $\mathcal{R}$-polar sets coincides with the class of null-sets of the Bessel capacity $\mathrm{Cap}_{2 / \alpha, \alpha^{\prime}}^{\partial}$. This implies that the condition

$$
\alpha<\frac{d+1}{d-1}
$$

holds if and only if the empty set is the only $\mathcal{R}$-polar set.

Condition (1.25) is satisfied if $\alpha=d=2$. Le Gall [13] succeeded in describing all solutions of the equation $\Delta u=u^{2}$ in the unit disk in $\mathbb{R}^{2}$. He established a 1-1 correspondence between all solutions and all pairs $(\Gamma, \nu)$, where $\Gamma$ is a closed subset of $\partial E$ and $\nu$ is a Radon measure on $\partial E \backslash \Gamma$. The solution corresponding to $(\Gamma, \nu)$ is expressed in terms of the Brownian snake - a path-valued Markov process introduced in his earlier publications (this process is closely related to the superBrownian motion). In [15], the results announced in [13] are proved in detail and are extended to a general smooth domain in $\mathbb{R}^{2}$. Recently Le Gall [16] extended some of the results of the present paper to a parabolic equation $\partial u / \partial t=\Delta u-u^{2}$ in a cylinder $\mathbb{R}_{+} \times E$. He defined the trace of a positive solution $u$ on $\{0\} \times E$ and

\footnotetext{
${ }^{5}$ In the particular case $\alpha=2$, the conjecture was confirmed earlier by Le Gall [14].
} 
proved propositions similar to our Theorems 1.3 and 1.4 [which he cited referring to our preprint].

A boundary value problem of type (1.22) was first considered by Gmira and Véron [12]. They investigated by purely analytic methods a class of functions $\psi$ such that the problem

$$
\Delta u=\psi(u) \text { in } E, \quad u=\nu \quad \text { on } \partial E
$$

is solvable for every finite measure $\nu$. This class contains $\psi(u)=u^{\alpha}$ with $\alpha$ subject to condition (1.23).

Marcus and Véron [17] investigated the equation $\Delta u=u^{\alpha}, \alpha>1$, in the unit $d$-dimensional ball. ${ }^{6}$ For every positive solution $u$ they define the trace $(\Gamma, \nu)$ of $u$ in terms of the boundary behavior of $u$.

Under condition (1.23), every pair (closed set $\Gamma$, Radon measure $\nu$ on $\partial E \backslash \Gamma$ ) is the trace of the uniquely defined positive solution $u$. (In the case $\alpha=d=2$, this was proved earlier by Le Gall.) More results in the same direction are announced in [18]. In particular, the existence of a solution is stated, for $\alpha \geq \frac{d+1}{d-1}$, under conditions stronger than conditions (i)-(iv) in our Theorems 1.3 and 1.4. The authors have also investigated the parabolic case in a setting similar to those of [16].

Added in proof. The results announced in [17] and [18] are proved in two recent preprints [M. Marcus and L. Véron, The boundary trace of positive solutions of semilinear elliptic equations, I: The subcritical case; II: The supercritical case, Université François Rabelais, Tours]. In addition, they give necessary and sufficient conditions for a pair $(\Gamma, \nu)$ to be a trace in terms of a class of exceptional sets on the boundary and they extend the characterization of exceptional sets, given in [6] for $\alpha \leq 2$, to the case $\alpha>2$. [Their method does not work for $\alpha \leq 2$.]

\section{Equation $L u=u^{\alpha}$. Operators $V_{D}$}

2.1. Class $\mathcal{U}(D)$. We denote by $\mathcal{U}(D)$ the class of all positive functions $u$ on $D$ such that

$$
L u=u^{\alpha} \quad \text { in } D
$$

and we put $\mathcal{U}=\mathcal{U}(E)$. We use the following facts:

2.1.A. (Comparison principle) If $D$ is a bounded open set and if $u, v \geq 0$ satisfy the conditions

$$
L u-u^{\alpha} \geq L v-v^{\alpha} \quad \text { in } D
$$

and

$$
\limsup _{x \rightarrow a, x \in D}[u(x)-v(x)] \leq 0 \quad \text { for all } a \in \partial D,
$$

then $u \leq v$ in $D$.

(See, e.g., Theorem 0.5 in [2].)

2.1.B. Suppose that $u_{n} \in \mathcal{U}(D)$ converge pointwise in $D$ to $u$. Then $u \in \mathcal{U}(D)$. Let $O$ be a relatively open subset of $\partial D$ and let $f$ be a continuous function on $O$. If all points of $O$ are regular and if $u_{n}=f$ on $O$, then $u=f$ on $O$. (See Theorem 1.2 in [3].)

\footnotetext{
${ }^{6}$ We emphasize that they cover the case $\alpha>2$, which can not be investigated by using superdiffusions
} 
2.1.C. Let $X_{D}$ be the exit measure of an $(L, \alpha)$-superdiffusion $X$ from an open set $D$. Then, for every Borel function $f: \partial D \rightarrow[0, \infty]$,

$$
u(x)=-\log P_{x} e^{-\left\langle f, X_{D}\right\rangle}
$$

belongs to $\mathcal{U}(D)$, and $u=f$ at every regular point $a \in \partial D$ where $f$ is continuous. If $D$ is regular and if $f$ is continuous on $\partial D$, then $u$ is the minimal element of $\mathcal{U}(D)$ such that $u=f$ on $\partial D$. If, in addition, $D$ is bounded and $f$ is finite, then $u$ is the unique element of $\mathcal{U}(D)$ such that $u=f$ on $\partial D$.

(See Theorems 1.2, 1.3 in [3] or Section II.3 in [4].)

2.1.D. (The mean value property) If $u \in \mathcal{U}(E)$ and if $\bar{D} \subset E$, then

$$
u(x)=-\log P_{x} e^{-\left\langle u, X_{D}\right\rangle} \quad \text { in } D .
$$

(See Lemma 1.1 in [3] or Theorem 2.3 in [6].)

2.1.E. Suppose that $D_{1} \supset D_{2}$ and $\Gamma \cap D_{1}=\emptyset$. Then $X_{D_{1}}(\Gamma) \geq X_{D_{2}}(\Gamma) P_{\mu}$-a.s. for every $\mu$.

(See Lemma 3.1 in [8].)

2.2. Operators $V_{D}$. With every open $D \subset E$ we associate an operator which acts on positive Borel functions by the formula

$$
V_{D}(f)(x)=-\log P_{x} e^{-\left\langle f_{E}, X_{D}\right\rangle},
$$

where $f_{E}=1_{E} f$. If $x \notin D$, then $P_{x}\left\{X_{D}=\delta_{x}\right\}=1$ and therefore $V_{D}(f)=f$ on $E \backslash D$. For every positive Borel $f, V_{D}(f) \in \mathcal{U}(D)$. [This follows, for instance, from Theorems 2.1-2.2 in [6].] Formula (1.8) implies that

$$
V_{D}(f) \leq K_{D} f .
$$

The operators $V_{D}$ have also the following properties:

2.2.A. $V_{D}\left(f_{1}\right) \leq V_{D}\left(f_{2}\right)$ for $f_{1} \leq f_{2}$.

2.2.B. If $D_{1} \subset D_{2}$, then $V_{D_{1}} V_{D_{2}}=V_{D_{2}}=V_{D_{2}} V_{D_{1}}$.

Proof. Put $V_{i}=V_{D_{i}}$ and $v_{i}=V_{i}(f)$. By the Markov property (1.11),

$$
v_{2}(x)=-\log P_{x} P_{X_{D_{1}}} e^{-\left\langle f, X_{D_{2}}\right\rangle}=-\log P_{x} e^{-\left\langle v_{2}, X_{D_{1}}\right\rangle}=V_{1}\left(v_{2}\right)(x) .
$$

The equality $V_{2}\left(v_{1}\right)=v_{2}$ holds because $v_{1}=f$ on $E \backslash D_{1}$, and $V_{2}(g)$ depends only on the values of $g$ on $E \backslash D_{2}$.

2.2.C. For every $f_{1}, f_{2}$,

$$
V_{D}\left(f_{1}+f_{2}\right) \leq V_{D}\left(f_{1}\right)+V_{D}\left(f_{2}\right) .
$$

Proof. Let $u_{i}=V_{D}\left(f_{i}\right), i=1,2$, and $u=V_{D}\left(f_{1}+f_{2}\right)$. By (1.8),

$$
u_{i}+\mathcal{E}_{D}\left(u_{i}\right)=K_{D} f_{i}, \quad u+\mathcal{E}(u)=K_{D} f_{1}+K_{D} f_{2} .
$$

We have $\left(u_{1}+u_{2}\right)^{\alpha}=u_{1}^{\alpha}+u_{2}^{\alpha}+\varphi$, where $\varphi \geq 0$. Note that $u_{1}+u_{2}+\mathcal{E}\left(u_{1}+u_{2}\right)=$ $u_{1}+u_{2}+\mathcal{E}\left(u_{1}\right)+\mathcal{E}\left(u_{2}\right)+G_{D} \varphi=K_{D}\left(f_{1}+f_{2}\right)+G_{D} \varphi=u+\mathcal{E}(u)+G_{D} \varphi$. By Theorem 2.1 in [8], this implies $u_{1}+u_{2} \geq u$.

2.2.D. For every $D_{1}, D_{2}$,

$$
V_{D_{1} \cap D_{2}}(f) \leq V_{D_{1}}(f)+V_{D_{2}}(f) \text { in } D_{1} \cap D_{2} .
$$


Proof. Put $D=D_{1} \cap D_{2}$. Note that $\partial D=B_{1} \cup B_{2}$, where $B_{1} \subset \partial D_{1}, B_{2} \subset \partial D_{2}$ and $B_{1} \cap B_{2}=\emptyset$. Put $f_{i}=1_{B_{i}} f$. We have $f=f_{1}+f_{2}$ on $\partial D$, and therefore $V_{D}(f)=V_{D}\left(f_{1}+f_{2}\right)$ on $D$. By 2.2.C,

$$
V_{D}(f) \leq V_{D}\left(f_{1}\right)+V_{D}\left(f_{2}\right) \text { on } D \text {. }
$$

By 2.1.E, $X_{D}(\Gamma) \leq X_{D_{1}}(\Gamma)$ for all $\Gamma \subset B_{1}$. Hence

$$
\left\langle f_{1}, X_{D}\right\rangle=\int_{B_{1}} f d X_{D} \leq \int_{B_{1}} f d X_{D_{1}} \leq\left\langle f, X_{D_{1}}\right\rangle
$$

which implies $V_{D}\left(f_{1}\right) \leq V_{D_{1}}(f)$. Analogously, $V_{D}\left(f_{2}\right) \leq V_{D_{2}}(f)$, and (2.8) implies 2.2.D.

\subsection{Action of $V_{D}$ on $\mathcal{U}$.}

Theorem 2.1. Suppose that $D$ is a regular open subset of $E$ and $u \in \mathcal{U}$. Then

$$
V_{D}(u) \leq u \text {. }
$$

$$
\text { If } D_{1} \subset D_{2} \text {, then }
$$

$$
V_{D_{2}}(u) \leq V_{D_{1}}(u) .
$$

Proof. Let $v=V_{D}(u)$. Note that $\partial D=A \cup B$, where $A=\partial D \cap \partial E$ and $B=\partial D \cap E$. Denote by $A_{0}$ the set of all points $a \in A$ at a positive distance from $B$. By 2.1.C, $v=u$ on $B$ and $v=0$ on $A_{0}$. Consider a sequence of continuous functions $f_{n}$ on $\partial D$ such that $f_{n} \uparrow u$ on $B$ and $f_{n}=0$ on $A$. Let $v_{n}(x)=-\log P_{x} e^{-\left\langle f_{n}, X_{D}\right\rangle}$. By 2.1.C, $v_{n} \in \mathcal{U}(D)$ and $v_{n}=f_{n}$ on $\partial D$. Therefore

$$
\limsup _{x \rightarrow a, x \in D}\left[v_{n}(x)-u(x)\right] \leq 0
$$

for all $a \in \partial D$. By 2.1.A, $v_{n} \leq u$, and (2.9) holds because $v_{n} \uparrow v$.

If $D_{1} \subset D_{2}$, then, by 2.2.B, (2.9) and 2.2.A, $V_{D_{2}}(u)=V_{D_{1}} V_{D_{2}}(u) \leq V_{D_{1}}(u)$.

\section{Operators $Q_{B}$}

3.1. Construction. For every closed set $B \subset \partial E$ and for every $\varepsilon>0$ we put

$$
D(B, \varepsilon)=\{x \in E: d(x, B)>\varepsilon\} .
$$

The open sets $(3.1)$ are regular. If $u \in \mathcal{U}$, then by Theorem 2.1, $V_{D(B, \varepsilon)}(u)$ is monotone increasing in $\varepsilon$ and therefore there exists a limit

$$
Q_{B}(u)=\lim _{\varepsilon \rightarrow 0} V_{D(B, \varepsilon)}(u) .
$$

It follows from 2.1.B that $Q_{B}(u) \in \mathcal{U}$. The operators $Q_{B}$ have the following properties:

3.1.A. $Q_{B}\left(u_{1}\right) \leq Q_{B}\left(u_{2}\right)$ for $u_{1} \leq u_{2}$.

3.1.B. $Q_{B}(u) \leq u$.

3.1.C. For every $B_{1}, B_{2}, \quad Q_{B_{1} \cup B_{2}}(u) \leq Q_{B_{1}}(u)+Q_{B_{2}}(u)$.

3.1.D. $Q_{\partial E}(u)=u$.

3.1.E. If $u \leq u_{1}+u_{2}$, then $Q_{B}(u) \leq Q_{B}\left(u_{1}\right)+Q_{B}\left(u_{2}\right)$.

Property 3.1.A is an implication of 2.2.A; 3.1.B follows from (2.9); 3.1.C is an implication of 2.2.D and the relation $D\left(B_{1} \cup B_{2}, \varepsilon\right)=D\left(B_{1}, \varepsilon\right) \cap D\left(B_{2}, \varepsilon\right)$. 3.1.D holds because, by 2.1.D, $V_{D(\partial E, \varepsilon)}(u)=u$ for all $\varepsilon$. Finally, 3.1.E follows from 2.2.C and 2.2.A. 


\subsection{Extremal characterization.}

Theorem 3.1. For every $u \in \mathcal{U}$, the function $u_{B}=Q_{B}(u)$ is the maximal element of $\mathcal{U}$ subject to the conditions

$$
u_{B} \leq u ; \quad u_{B}=0 \quad \text { on } \partial E \backslash B .
$$

Proof. Put $D^{\varepsilon}=D(B, \varepsilon), B^{\varepsilon}=\partial D_{\varepsilon} \cap E$ and denote by $A^{\varepsilon}$ the set of all $x \in \partial E$ at a distance $>\varepsilon$ from $B$. By 2.1.C, the function $v^{\varepsilon}=V_{D^{\varepsilon}}(u)$ belongs to $\mathcal{U}\left(D^{\varepsilon}\right)$ and satisfies the conditions $v^{\varepsilon}=0$ on $A^{\varepsilon}, v^{\varepsilon}=u$ on $B^{\varepsilon}$. It follows from 3.1.B, (3.2) and 2.1.B that $u_{B}$ belongs to $\mathcal{U}$ and satisfies (3.3).

Suppose (3.3) holds for $\tilde{u}$. Then $\tilde{u} \leq u=v^{\varepsilon}$ on $B^{\varepsilon}$ and $\tilde{u}=0 \leq v^{\varepsilon}$ on $\partial D^{\varepsilon} \backslash B^{\varepsilon} \subset \partial E \backslash B$. By 2.1.A, $\tilde{u} \leq v^{\varepsilon}$ in $D^{\varepsilon}$, and therefore $\tilde{u} \leq u_{B}$.

Theorem 3.1 implies:

3.2.A. If $B_{1} \supset B_{2}$, then $Q_{B_{1}} Q_{B_{2}}=Q_{B_{2}}=Q_{B_{2}} Q_{B_{1}}$ and $Q_{B_{1}}(u) \geq Q_{B_{2}}(u)$.

3.2.B. If $B$ and $B_{0}$ are disjoint compact subsets of $\partial E$, then $Q_{B} Q_{B_{0}}=0$.

Indeed, $Q_{B}\left[Q_{B_{0}}(u)\right]=0$ on $\partial E \backslash B$ and $Q_{B}\left[Q_{B_{0}}(u)\right] \leq Q_{B_{0}}(u)=0$ on $\partial E \backslash B_{0}$.

3.3. Probabilistic representation. We say that a sequence of open sets $D_{n}$ is a standard sequence approximating $E$ if their closures $\bar{D}_{n}$ are compact, $\bar{D}_{n} \subset D_{n+1}$ and $D_{n} \uparrow E$. It is proved in Section II.5 of [4] that, for every $u \in \mathcal{U}$, there exists a function $Z$ such that

$$
Z=\lim \left\langle u, X_{D_{n}}\right\rangle \quad P_{\mu} \text {-a.s. }
$$

for every standard sequence $D_{n}$ and every $\mu \in \mathcal{M}(E)$. Since $V_{D_{n}}(u)=u$ in $D_{n}$, the dominated convergence theorem implies that

$$
u(x)=-\log P_{x} e^{-Z} .
$$

By applying this result to $u_{B}=Q_{B}(u)$, we get a probabilistic representation of the operators $Q_{B}$ :

$$
Q_{B}(u)(x)=-\log P_{x} e^{-Z_{B}},
$$

where

$$
Z_{B}=\lim \left\langle u_{B}, X_{D_{n}}\right\rangle \quad P_{\mu} \text {-a.s. }
$$

It follows from 3.2.A and 3.1.C that

3.3.A. If $B_{1} \supset B_{2}$, then $Z_{B_{1}} \geq Z_{B_{2}}$ a.s.

3.3.B. For every $B_{1}, B_{2}, Z_{B_{1} \cup B_{2}} \leq Z_{B_{1}}+Z_{B_{2}}$ a.s.

We need another representation of $Z_{B}$ :

3.3.C. ${ }^{7}$ For every sequence $\varepsilon_{n} \downarrow 0$ and every $\mu \in \mathcal{M}(E)$,

$$
Z_{B}=\lim \left\langle u, X_{D\left(B, \varepsilon_{n}\right)}\right\rangle \quad P_{\mu} \text {-a.s. }
$$

Proof. $1^{\circ}$. Put $\tilde{D}_{n}=D\left(B, \varepsilon_{n}\right), Y_{n}=e^{-\left\langle u, X_{\tilde{D}_{n}}\right\rangle}$. By (1.11), (1.7), (2.6) and (2.9),

$$
P_{\mu}\left\{Y_{n+1} \mid \mathcal{F}_{\subset \tilde{D}_{n}}\right\}=P_{X_{\tilde{D}_{n}}} Y_{n+1}=e^{-\left\langle V_{\tilde{D}_{n+1}}(u), X_{\tilde{D}_{n}}\right\rangle} \geq Y_{n} \quad P_{\mu} \text {-a.s. }
$$

Hence $\left(Y_{n}, \mathcal{F}_{\subset \tilde{D}_{n}}, P_{\mu}\right)$ is a bounded submartingale which implies the existence, a.s., of the limit

$$
\tilde{Z}_{B}=\lim \left\langle u, X_{\tilde{D}_{n}}\right\rangle
$$

\footnotetext{
${ }^{7} \mathrm{By}\left\langle u, X_{D}\right\rangle$ we mean the integral of $u$ over $D^{c} \cap E$. In other words, we set $u=0$ on $\partial E$.
} 
By (3.2) and the dominated convergence theorem,

$$
P_{\mu} e^{-\tilde{Z}_{B}}=e^{-\left\langle u_{B}, \mu\right\rangle} .
$$

$2^{\circ}$. By $1^{\circ}$ applied to $u_{B}$, we get the existence of the limit

$$
\hat{Z}_{B}=\lim \left\langle u_{B}, X_{\tilde{D}_{n}}\right\rangle
$$

and the equation

$$
P_{\mu} e^{-\hat{Z}_{B}}=e^{-\left\langle Q_{B}\left(u_{B}\right), \mu\right\rangle} .
$$

By 3.2.A, $Q_{B}\left(u_{B}\right)=u_{B}$, and (3.12) and (3.10) yield

$$
P_{\mu} e^{-\hat{Z}_{B}}=P_{\mu} e^{\tilde{Z}_{B}}
$$

By 3.1.B, $\hat{Z}_{B} \leq \tilde{Z}_{B}$, and (3.13), (3.11) imply

$$
\tilde{Z}_{B}=\hat{Z}_{B}=\lim \left\langle u_{B}, X_{\tilde{D}_{n}}\right\rangle \text { a.s. }
$$

$3^{\circ}$. Fix $\varepsilon>0$. By (3.3), $u_{B}=0$ on $\partial E \backslash B$. Therefore there exists $\delta_{n}>0$ such that $u_{B}(x) \leq \varepsilon$ if $x \in \tilde{D}_{n}$ and $d(x, \partial E) \leq \delta_{n}$. Formula (3.7) holds for a standard sequence $D_{n}=\left\{x: d(x, B)>\varepsilon_{n}, d(x, \partial E)>\delta_{n}\right\}$ approximating $E$. We have $D_{n}=D_{n}^{*} \cap \tilde{D}_{n}$, where $D_{n}^{*}=\left\{x: d(x, \partial E)>\delta_{n}\right\}$, and, by 2.1.E,

$$
\left\langle u_{B}, X_{D_{n}}\right\rangle \leq\left\langle u_{B}, X_{\tilde{D}_{n}}\right\rangle+\left\langle\varepsilon, X_{D_{n}^{*}}\right\rangle .
$$

It follows from (3.15), (3.7) and (3.14) that

$$
Z_{B} \leq \tilde{Z}_{B}+\varepsilon \lim \left\langle 1, X_{D_{n}^{*}}\right\rangle .
$$

By [7, Lemma 1.2], for every $L$-harmonic function $h$ and for every standard sequence $D_{n}$, there exists, $P_{\mu}$-a.s., a finite $\operatorname{limit} \lim \left\langle h, X_{D_{n}}\right\rangle$, and the limit does not depend, $P_{\mu}$-a.s., on $D_{n}$. This is applicable to $h=1$ and $D_{n}=D_{n}^{*}$; and, by letting $\varepsilon \rightarrow 0$ in (3.16), we get $Z_{B} \leq \tilde{Z}_{B}$. Equations (3.6) and (3.10) imply $Z_{B}=\tilde{Z}_{B}$.

3.4. Trace of a moderate solution. It follows from Theorems 1.3, 2.1 and 3.1 in [7] and Theorem 1.2a in [6] that:

3.4.A. If $u$ is moderate, then

$$
h=u+\mathcal{E}(u)
$$

is the minimal $L$-harmonic majorant of $u$, and $u$ is the maximal solution dominated by $h$.

3.4.B. Let $h$ be an $L$-harmonic function with trace $\nu$. Equation (3.17) has a solution $u$ if and only if $\nu$ does not charge $\mathcal{R}$-polar sets.

3.4.C. If (3.17) holds for an $L$-harmonic $h$, then $u \in \mathcal{U}$.

The trace of a moderate solution $u$ is defined as the trace of the $L$-harmonic function $h=u+\mathcal{E}(u)$. By 3.4.B, a finite measure $\nu$ is a trace if and only if $\nu(B)=0$ for all $\mathcal{R}$-polar sets $B$. It follows from 3.4.A that the inequality $u_{1} \leq u_{2}$ between two moderate solutions is equivalent to the inequality $h_{1} \leq h_{2}$ between their minimal $L$-harmonic majorants, which is equivalent to the inequality $\nu_{1} \leq \nu_{2}$ between the traces.

We use the following facts. Let $k$ be the Poisson kernel in $E$. If $h$ is a positive $L$-harmonic function with trace $\nu$, then there exists a measure $\Pi_{x}^{h}$ on the space of paths killed at the first exit from $E$ such that, for every $B \subset \partial E$,

$$
\Pi_{x}^{h}\left\{\xi_{\zeta} \in B\right\}=\int_{B} k(x, y) \nu(d y)
$$


and, for every stopping time $\tau$,

$$
\Pi_{x}^{h}\{\tau<\zeta\}=\Pi_{x} h\left(\xi_{\tau}\right) .
$$

[The probability measure $\frac{1}{h(x)} \Pi_{x}^{h}$ is called the $h$-transform of $\Pi_{x}$.] Moreover,

$$
\Pi_{x}^{h}=\int \Pi_{x}^{y} \nu(d y)
$$

where $\Pi_{x}^{y}$ is the measure corresponding to the $L$-harmonic function $k(\cdot, y)$.

Theorem 3.2. If $u$ is a moderate solution, then, for every $B, u_{B}=Q_{B}(u)$ is also moderate and the trace of $u_{B}$ is the restriction of the trace of $u$ to $B$.

Proof. The first part follows from 3.1.B.

Let $\nu$ be the trace of $u$. Consider the minimal $L$-harmonic majorant

$$
h(x)=\int_{\partial E} k(x, y) \nu(d y)
$$

of $u$ and the minimal $L$-harmonic majorant $h_{B}$ of $u_{B}$. Put

$$
\hat{h}_{B}(x)=\int_{B} k(x, y) \nu(d y) .
$$

By (3.18) and (3.19),

$$
\hat{h}_{B}(x)=\Pi_{x}^{h}\left\{\xi_{\zeta} \in B\right\}=\lim _{\varepsilon \rightarrow 0} \Pi_{x}^{h}\left\{\tau_{\varepsilon}<\zeta\right\}=\lim _{\varepsilon \rightarrow 0} \Pi_{x} h\left(\xi_{\tau_{\varepsilon}}\right),
$$

where $\tau_{\varepsilon}$ is the first exit time from $D(B, \varepsilon)$. By $(2.7)$,

$$
V_{D(B, \varepsilon)}(u)(x) \leq \Pi_{x} u\left(\xi_{\tau_{\varepsilon}}\right) \leq \Pi_{x} h\left(\xi_{\tau_{\varepsilon}}\right) .
$$

By $(3.2),(3.21)$ and $(3.22), u_{B} \leq \hat{h}_{B}$, which implies

$$
h_{B} \leq \hat{h}_{B}
$$

By 3.4.B, there exists $\hat{u}_{B}$ such that $\hat{u}_{B}+\mathcal{E}\left(\hat{u}_{B}\right)=\hat{h}_{B}$. Note that $\hat{u}_{B} \leq u$ and $\hat{u}_{B} \leq \hat{h}_{B}=0$ on $\partial E \backslash B$. By Theorem 3.1, $u_{B} \geq \hat{u}_{B}$, and therefore $h_{B} \geq \hat{h}_{B}$. By $(3.23), h_{B}=\hat{h}_{B}$.

3.5. General definition of trace. Fix a solution $u$. We say that a compact set $B \subset \partial E$ is moderate for $u$ if the solution $u_{B}=Q_{B}(u)$ is moderate. Let $\nu_{B}$ stand for the trace of $u_{B}$. By 3.1.C, the union of two moderate sets is moderate. Suppose that $B$ is moderate and let $\tilde{B} \subset B$. By 3.2.A, $u_{\tilde{B}} \leq u_{B}$ and therefore $\tilde{B}$ is moderate. It follows from Theorem 3.2 and 3.2.A that $\nu_{\tilde{B}}$ is the restriction of $\nu_{B}$ to $\tilde{B}$.

A relatively open subset $A$ of $\partial E$ is called moderate if all compact subsets of $A$ are moderate. The union $O$ of all moderate open sets is moderate. Clearly, there exists a unique measure $\nu$ on $O$ such that its restriction to an arbitrary compact subset $B$ coincides with $\nu_{B}$. This measure is a Radon measure on $O$ (that is, it is finite on all compact subsets). We call the closed set $\Gamma=\partial E \backslash O$ the singular set of the solution $u$ and we call the pair $(\Gamma, \nu)$ the trace of $u$ on $\partial E$. A solution $u$ is moderate if and only if the singular set is empty (in this case $\nu(\partial E)<\infty$ ).

If a compact set $B$ is $\mathcal{R}$-polar, then $Q_{B}(u)=0$. This follows from the relation

$$
\{\mathcal{R} \cap B=\emptyset\}=\bigcup_{\varepsilon}\left\{X_{D(B, \varepsilon)}=0\right\}
$$


(see, e.g., the proof of Theorem 4.2 in [4]). Hence $\mathcal{R}$-polar sets are moderate for all $u$. We conclude from 3.4.A,B,C that:

3.5.A. If $(\Gamma, \nu)$ is the trace of a solution $u$, then $\nu$ does not charge $\mathcal{R}$-polar sets.

\section{Solutions Determined By CONTINuous lineAR ADDitive FUnCTiOnAls}

4.1. Continuous linear additive functionals. Let $X$ be a superdiffusion in $E$ and let $\mathcal{M}^{*}$ be a set of finite measures on $E$ which contains all Dirac measures $\delta_{x}, x \in E$. We assume that $\mathcal{M}^{*}$ contains, with every $\mu$, all measures $\tilde{\mu} \leq \mu$ and that $P_{\mu}\left\{X_{D} \in \mathcal{M}^{*}\right\}=1$ for all $D$ and all $\mu \in \mathcal{M}^{*}$. Denote by $\mathcal{F}$ the $\sigma$-algebra generated by $X_{s}, s<\infty$, and by $\mathcal{F}_{t}$ the $\sigma$-algebra generated by $X_{s}, s \leq t$. A function $A_{t}(\omega)$ from $[0, \infty] \times \Omega$ to $[0, \infty)$ is called a finite continuous additive functional of $X$ with determining set $\mathcal{M}^{*}$ if:

4.1.A. $A_{0}=0$.

4.1.B. For every $t$ and every $\mu, A_{t}$ is measurable with respect to the $P_{\mu}$-completion of $\mathcal{F}$; it is also measurable with respect to $P_{\mu}$-completion of $\mathcal{F}_{t}$ if $\mu \in \mathcal{M}^{*}$.

4.1.C. $A_{s+t}=A_{s}+\theta_{s} A_{t} P_{\mu}$-a.s. for all $\mu \in \mathcal{M}^{*}$ and all pairs $s$, t. (Here $\theta_{s}$ are the shift operators for $X$.)

4.1.D. $A_{t}$ is continuous in $t$ for $P_{\mu}$-almost all $\omega$ for every $\mu \in \mathcal{M}^{*}$.

A continuous additive functional with determining set $\mathcal{M}^{*}$ is a function $A_{t}(\omega)$ which can be represented as the sum of a countable family of finite continuous additive functionals with the same determining set. [If $\sigma=\sup \left\{t: A_{t}<\infty\right\}$, then $A_{t}$ is continuous on $[0, \sigma)$ and $A_{t}=\infty$ for $t>\sigma$.] To every functional $A_{t}$ there corresponds a diffuse measure ${ }^{8} a(d t)$ on $(0, \infty]$ such that $a(0, t]=A_{t}$ for all $t$. This measure is finite if and only the functional $A$ is finite.

If $A^{n}$ are continuous additive functionals with determining sets $\mathcal{M}_{n}^{*}$ and if $\mathcal{M}^{*} \subset$ $\mathcal{M}_{n}^{*}$ for all $n$, then $A_{t}=A_{t}^{1}+\cdots+A_{t}^{n}+\ldots$ is a continuous additive functional with determining set $\mathcal{M}^{*}$.

Let $A$ and $\tilde{A}$ be continuous additive functionals with determining sets $\mathcal{M}^{*}$ and $\tilde{\mathcal{M}}^{*}$. We say that $A$ and $\tilde{A}$ are equivalent, and write $A \sim \tilde{A}$, if $P_{\mu}\left\{A_{t}=\tilde{A}_{t}\right\}=1$ for all $t$ and all $\mu \in \mathcal{M}^{*} \cap \tilde{\mathcal{M}}^{*}$. We say that $A$ and $\tilde{A}$ are indistinguishable if, in addition, $\mathcal{M}^{*}=\tilde{\mathcal{M}}^{*}$.

We call

$$
h(x)=P_{x} A_{\infty}
$$

the potential and

$$
u(x)=-\log P_{x} e^{-A_{\infty}}
$$

the log-potential of $A$. We say that a continuous additive functional $A$ is linear if

$$
P_{\mu} A_{\infty}=\langle h, \mu\rangle
$$

and

$$
P_{\mu} e^{-A_{\infty}}=e^{-\langle u, \mu\rangle}
$$

for all $\mu$ in the determining set $\mathcal{M}^{*}$.

\footnotetext{
${ }^{8} \mathrm{~A}$ measure $a$ is called diffuse if it does not charge single points.
} 
Suppose that the potential $h$ of a continuous linear additive (CLA) functional $A$ is an $L$-harmonic function $h$. It follows from Theorem 1.2 in [9] that the logpotential $u$ of $A$ satisfies the equation

$$
u+\mathcal{E}(u)=h \quad \text { in } E .
$$

Moreover, by Theorem 2.1 in [8], $u$ is the unique solution of (4.5). By 3.4.C, $u$ satisfies

$$
L u=u^{\alpha} \quad \text { in } E .
$$

Clearly, $u$ is a moderate solution of (4.6).

4.2. Classes $H^{*}$ and $H^{* *}$. Denote by $H^{*}$ the class of all positive $L$-harmonic functions $h$ such that equation (4.5) has a positive solution $u$. Put $h \in H^{* *}$ if $h \in H^{*}$ and if $\mathcal{E}(h)(x)<\infty$ for some $x$ (which implies that $\mathcal{E}(h)(x)<\infty$ for all $x$ ). It follows from [7, Theorem 3.1 and proof of Theorem 2.2] (cf. $[9,4.1 . B]$ ) that every $h \in H^{*}$ can be represented in the form

$$
h=h_{1}+\cdots+h_{n}+\ldots \quad \text { with } h_{n} \in H^{* *} .
$$

Theorems 1.7, 1.1, 1.3 in [10] imply

Theorem 4.1. If $h \in H^{* *}$, then there exists a unique (up to indistinguishability) continuous linear additive functional $A$ of $X$ with potential $h$ and determining set $\{\mu:\langle h+\mathcal{E}(h), \mu\rangle<\infty\}$. The log-potential $u$ of $A$ is determined uniquely by (4.5).

Remark. If $A^{n}$ are CLA functionals corresponding to $h_{n} \in H^{* *}$ by Theorem 4.1 and if $h=h_{1}+\cdots+h_{n}+\cdots \in H^{* *}$, then $A^{1}+\cdots+A^{n}+\ldots$ is equivalent to the CLA functional corresponding to $h$.

4.3. Continuous linear additive functional with spectral measure $\nu$. Denote by $\mathcal{N}^{*}$ the set of all measures $\nu$ on $\partial E$ such that $K \nu \in H^{*}$. Analogously, $\nu \in \mathcal{N}^{* *}$ if $K \nu \in H^{* *}$. Put $\nu \in \mathcal{N}$ if there exist $\nu_{n} \in \mathcal{N}^{* *}$ such that

$$
\nu=\nu_{1}+\cdots+\nu_{n}+\ldots
$$

Note that $\mathcal{N} \supset \mathcal{N}^{*}$.

Remark 1. Clearly, every $\nu \in \mathcal{N}$ is $\Sigma$-finite.

Remark 2. If $\nu^{n} \in \mathcal{N}^{* *}$ and $\nu^{n} \uparrow \nu \in \mathcal{N}^{* *}$, then, by the Remark to Theorem 4.1, $\lim A^{\nu_{n}} \sim A^{\nu}$.

Theorem 4.2. It is possible to define for every $\nu \in \mathcal{N}$ a continuous linear additive functional $A^{\nu}$ of $X$ with determining set $\mathcal{M}^{*}(\nu)$ in such a way that:

4.3.A. If $\nu=\nu_{1}+\cdots+\nu_{n}+\ldots$, then $A^{\nu}$ is equivalent to $A^{\nu_{1}}+\cdots+A^{\nu_{n}}+\ldots$

4.3.B. If $\nu \in \mathcal{N}^{* *}$ and if $h=K \nu$, then $A^{\nu}$ is equivalent to the CLA functional corresponding to $h=K \nu$ by Theorem 4.1.

4.3.C. For every $\nu \in \mathcal{N}$, the log-potential $u$ of $A^{\nu}$ satisfies the equation (4.6).

Proof. $1^{\circ}$. We use Theorem 4.1 to define $A^{\nu}$ for $\nu \in \mathcal{N}^{* *}$, and we define $A^{\nu}$ as the sum of $A^{\nu_{n}}$ if $\nu$ is defined by (4.8). To justify this definition, we prove that, if $\nu=\sum \nu_{i}=\sum \tilde{\nu}_{j}$, then $\sum A^{\nu_{i}} \sim \sum A^{\tilde{\nu}_{j}}$. Indeed, since the measure $\nu$ is $\Sigma$-finite, there exists a finite measure $\mu$ such that $\nu(d x)=\rho(x) \mu(d x)$ for a positive function $\rho$. Put

$$
\nu^{m}=\nu_{1}+\cdots+\nu_{m}, \quad \tilde{\nu}^{n}=\tilde{\nu}_{1}+\cdots+\tilde{\nu}_{n} .
$$


By the Radon-Nikodým theorem, $\nu^{m}(d x)=\rho^{m}(x) \mu(d x), \tilde{\nu}^{n}(d x)=\tilde{\rho}^{n}(x) \mu(d x)$ with $\lim \rho^{m}=\lim \tilde{\rho}^{n}=\rho \mu$-a.e. The measures $\nu^{m n}(d x)=\left[\rho^{m}(x) \wedge \tilde{\rho}^{n}(x)\right] \mu(d x)$ belong to $\mathcal{N}^{* *}$. The corresponding functionals $A^{m n}=A^{\nu^{m n}}$ are monotone increasing in $m$ and $n$, and therefore

$$
\lim _{m} \lim _{n} A^{m n}=\lim _{n} \lim _{m} A^{m n}=\sup _{m, n} A^{m n} .
$$

Since $\nu^{m n} \uparrow \nu^{m}$ as $n \rightarrow \infty$, we have $\lim _{n} A^{m n} \sim A^{\nu^{m}}$ by Remark 2. Clearly, $A^{\nu^{m}} \sim A^{\nu_{1}}+\cdots+A^{\nu_{m}}$, and therefore the left side in (4.9) is equal to $\sum A^{\nu_{i}}$. Analogously, the right side is equal to $\sum \tilde{A}^{\nu_{j}}$.

$2^{\circ}$. Properties 4.3.A and 4.3.B hold for $\nu \in \mathcal{N}^{* *}$ by Theorem 4.1, and they can be obtained for $\nu \in \mathcal{N}$ by a passage to the limit.

$3^{\circ}$. If $\nu_{k} \in \mathcal{N}^{* *}$, then $\nu^{n}=\nu_{1}+\cdots+\nu_{n} \in \mathcal{N}^{* *}$ for all $n$, and the log-potential $u_{n}$ of $A^{\nu}$ satisfies (4.4) by Theorem 4.1 and (4.6) by 3.4.C. The function $u=\lim u_{n}$ satisfies (4.6) by 2.1.B. We get (4.4) by a passage to the limit.

We call $A^{\nu}$ the CLA functional with spectral measure $\nu$.

Theorem 4.3. A $\Sigma$-finite measure $\nu$ belongs to $\mathcal{N}$ if and only if it charges no $\mathcal{R}$-polar sets.

Proof. For a finite measure $\nu$, this follows from 3.4.B. The extension to $\Sigma$-finite $\nu$ is obvious.

Theorem 4.4. Let $\nu \in \mathcal{N}$. The following three conditions are equivalent:

(i) $\nu$ is finite;

(ii) the potential $h$ of $A^{\nu}$ is L-harmonic;

(iii) the log-potential $u$ of $A^{\nu}$ is a moderate solution of (4.6).

Proof. The equivalence of (i) and (ii) follows from the relation

$$
h(x)=P_{x} A_{\infty}^{\nu}=\int k(x, y) \nu(d y),
$$

which follows from 4.3.B if $\nu \in \mathcal{N}^{* *}$ and which can be obtained by a monotone passage to the limit for an arbitrary $\nu \in \mathcal{N}$.

Suppose that $\nu_{n} \in \mathcal{N}^{* *}$ and $\nu_{n} \uparrow \nu$. The potential $h_{n}$ and the log-potential $u_{n}$ of $A^{\nu_{n}}$ are related by equation $u_{n}+\mathcal{E}\left(u_{n}\right)=h_{n}$. Clearly, $h_{n} \uparrow h, u_{n} \uparrow u$, and therefore $u+\mathcal{E}(u)=h$. If $h$ is $L$-harmonic, then $u$ is moderate. On the other hand, if $u$ is dominated by an $L$-harmonic $\tilde{h}$, then $u_{n} \leq \tilde{h}$, which implies $K \nu_{n} \leq \tilde{h}$. We conclude that $K \nu \leq \tilde{h}$, and therefore $\nu$ is finite.

\subsection{Log-potentials of functionals $A^{\nu}$.}

4.4.A. The log-potential $u$ of $A^{\nu}$ vanishes on $\partial E$ outside the support of $\nu$.

Proof. Let $\nu(U)=0$ for an open subset $U$ of $\partial E$. If $\nu_{n}$ are defined by (4.8), then $\nu_{n}(U)=0$. We have $u_{n}+\mathcal{E}\left(u_{n}\right)=h_{n}$ where $h_{n}$ is the potential and $u_{n}$ is the logpotential of $A^{\nu_{n}}$. By (1.5), $h_{n}=0$ on $U$. Hence $u_{n}=0$ on $U$ and $u=\lim u_{n}=0$ on $U$ by 2.1 .B.

4.4.B. If $u_{1}, u_{2}, u$ are the log-potentials of functionals $A^{\nu_{1}}, A^{\nu_{2}}, A^{\nu_{1}+\nu_{2}}$, then $u \leq$ $u_{1}+u_{2}$. 
Proof. If $\nu_{1}, \nu_{2} \in \mathcal{N}^{* *}$, then $u_{i}+\mathcal{E}\left(u_{i}\right)=K \nu_{i}<\infty$ and $u+\mathcal{E}(u)=K\left(\nu_{1}+\nu_{2}\right)$. We get $u \leq u_{1}+u_{2}$ by the same computation as in proof of 2.2.C. The general case is covered by a monotone passage to the limit.

4.4.C. Suppose that $\nu \in \mathcal{N}$ is finite and is concentrated on a closed set $B \subset \partial E$. Let $h$ be the potential and $u$ be the log-potential of $A^{\nu}$. If $\varepsilon_{n} \downarrow 0$, then

$$
A_{\infty}^{\nu}=\lim \left\langle u, X_{D\left(B, \varepsilon_{n}\right)}\right\rangle \quad P_{\mu} \text {-a.s. }
$$

for all $\mu \in \mathcal{M}^{*}(\nu)$.

Proof. By Theorem 4.4, $u$ is a moderate solution with trace $\nu$. It follows from Theorem 3.2 that $u_{B}=Q_{B}(u)$ has the same trace as $u$, and therefore $u_{B}=u$. By 3.3.C,

$$
Z=\lim \left\langle u, X_{D_{n}}\right\rangle=\lim \left\langle u, X_{D\left(B, \varepsilon_{n}\right)}\right\rangle \quad \text { a.s. }
$$

for an arbitrary standard sequence $D_{n}$. By (4.5), $u \leq h$, and, by Theorem 5.1 in $[9]$,

$$
A_{\infty}^{\nu}=\lim \left\langle h, X_{D_{n}}\right\rangle \geq Z \quad P_{\mu} \text {-a.s. }
$$

if $\mu \in \mathcal{M}^{*}(\nu)$. By (3.5), and (4.2),

$$
u(x)=-\log P_{x} e^{-Z}=-\log P_{x} e^{-A_{\infty}^{\nu}} .
$$

Formula (4.10) follows from (4.11), (4.12) and (4.13).

4.4.D. Suppose that $\nu \in \mathcal{N}$ vanishes on a closed set $B$ and is finite on a neighborhood $U$ of $B$. If $u$ is the log-potential of $A^{\nu}$, then $Q_{B}(u)=0$.

Proof. Denote by $\nu_{1}, \nu_{2}$ the restrictions of $\nu$ to $U$ and $U^{c}$, respectively. Let $u_{1}$ and $u_{2}$ be the log-potentials of $A^{\nu_{1}}$ and $A^{\nu_{2}}$. By 4.4.B, $u \leq u_{1}+u_{2}$ and, by 3.1.E, $Q_{B}(u) \leq Q_{B}\left(u_{1}\right)+Q_{B}\left(u_{2}\right)$.

By Theorem 4.4, $u_{1}$ is a moderate solution. Its trace $\nu_{1}$ vanishes on $B$, and $Q_{B}\left(u_{1}\right)=0$ by Theorem 3.2.

By 4.4.A, $u_{2}=0$ on $U$, and we conclude from Theorem 3.1 that $Q_{B}\left(u_{2}\right)=0$.

\section{5. $(\Gamma, \nu)$-SOLUtions}

5.1. Let $O$ be an open set on the boundary $\partial E$ and let $\nu$ be a measure on $\partial E$ which charges no $\mathcal{R}$-polar set. We put $\nu \in \mathcal{N}(O)$ if $\nu$ is concentrated on $O$ and if its restriction to $O$ (which we denote again by $\nu$ ) is a Radon measure on $O$. All measures $\nu \in \mathcal{N}(O)$ are $\Sigma$-finite and, by Theorem 4.3, $\mathcal{N}(O) \subset \mathcal{N}$. Let $\Gamma$ be a closed subset of $\partial E$. By Theorem 2.1 in [3],

$$
w(x)=-\log P_{x}\{\mathcal{R} \cap \Gamma=\emptyset\}
$$

is the maximal element of $\mathcal{U}$ such that

$$
w=0 \quad \text { on } O=\partial E \backslash \Gamma .
$$

For every $\nu \in \mathcal{N}(O)$,

$$
u(x)=-\log P_{x}\left\{\mathcal{R} \cap \Gamma=\emptyset, e^{-A_{\infty}^{\nu}}\right\}
$$

is also an element of $\mathcal{U}$. [This is proved in Section 4 of [11] by using the probabilistic description of $\mathcal{U}$ given in Section 0.8 of [5].] We call $u$ the $(\Gamma, \nu)$-solution. Our objective is to evaluate the trace of such solution. To simplify notation, we drop 
the superscript $\infty$ in (5.3) and similar formulae. It follows from (5.3) and Theorem II.4.3 in [4] that

$$
P_{\mu}\left\{\mathcal{R} \cap \Gamma=\emptyset, e^{-A^{\nu}}\right\}=e^{-\langle u, \mu\rangle}
$$

if the support of the measure $\mu$ is disjoint from $\Gamma$.

\section{2 .}

Theorem 5.1. Let $u$ be the $(\Gamma, \nu)$-solution and let $\nu^{\prime}$ be the restriction of $\nu$ to a closed subset $B$ of $O$. Then $Q_{B}(u)$ is equal to the log-potential of $A^{\prime}=A^{\nu^{\prime}}$, and

$$
Z_{B}=A_{\infty}^{\prime} \quad P_{x} \text {-a.s. }
$$

for all $x$. (Here $Z_{B}$ is given by (3.7) or (3.8).)

Proof. Let $A^{\prime \prime}=A^{\nu^{\prime \prime}}$, where $\nu^{\prime \prime}$ is the restriction of $\nu$ to $B^{\prime \prime}=O \backslash B$. Denote by $u^{\prime}, u^{\prime \prime}$ the log-potentials of $A^{\prime}, A^{\prime \prime}$, and put $D_{\varepsilon}=D(B, \varepsilon)$. By $(2.6)$,

$$
u_{\varepsilon}(x)=V_{D_{\varepsilon}}(u)(x)=-\log P_{x} e^{-\left\langle u, X^{\varepsilon}\right\rangle},
$$

where $X^{\varepsilon}$ is the restriction to $E$ of the exit measure from $D_{\varepsilon}$. By (5.4), this implies

$$
u_{\varepsilon}(x)=-\log P_{x} P_{X^{\varepsilon}}\left\{\mathcal{R} \cap \Gamma=\emptyset, e^{-A^{\nu}}\right\} .
$$

By 4.4.C, $A^{\prime}$ is measurable with respect to the $P_{x}$-completion of $\mathcal{F}_{\supset D_{\varepsilon}}$; and, by (1.11), $P_{x} e^{-A^{\prime}}=P_{x} P_{X^{\varepsilon}} e^{-A^{\prime}}$. By (2.6) and (5.4),

$$
e^{-V_{D^{\varepsilon}}\left(u^{\prime \prime}\right)(x)}=P_{x} e^{-\left\langle u^{\prime \prime}, X^{\varepsilon}\right\rangle}=P_{x} P_{X^{\varepsilon}} e^{-A^{\prime \prime}} .
$$

By (5.4),

$$
P_{X^{\varepsilon}}\{\mathcal{R} \cap \Gamma \neq \emptyset\}=1-e^{-\left\langle w, X^{\varepsilon}\right\rangle} \leq\left\langle w, X^{\varepsilon}\right\rangle,
$$

where $w$ is given by (5.1). Hence,

$$
\begin{aligned}
\left|e^{-u^{\prime}(x)}-e^{-u_{\varepsilon}(x)}\right| \leq & \left|e^{-u^{\prime}(x)}-P_{x} P_{X_{\varepsilon}}\left\{\mathcal{R} \cap \Gamma=\emptyset, e^{-A^{\prime}}\right\}\right| \\
& +\left|P_{x} P_{X_{\varepsilon}}\left\{\mathcal{R} \cap \Gamma=\emptyset, e^{-A^{\prime}}\right\}-e^{-u_{\varepsilon}(x)}\right| \leq I_{\varepsilon}+J_{\varepsilon},
\end{aligned}
$$

where

$$
\begin{gathered}
I_{\varepsilon}=P_{x} P_{X^{\varepsilon}}\left\{\mathcal{R} \cap \Gamma=\emptyset, e^{-A^{\prime}}\left(1-e^{-A^{\prime \prime}}\right)\right\} \leq P_{x} P_{X^{\varepsilon}}\left(1-e^{-A^{\prime \prime}}\right)=1-e^{-V_{D^{\varepsilon}}\left(u^{\prime \prime}\right)(x)}, \\
J_{\varepsilon}=P_{x} P_{X^{\varepsilon}}\left\{\mathcal{R} \cap \Gamma \neq \emptyset, e^{-A^{\prime}}\right\} \leq 1-P_{x} e^{-\left\langle w, X^{\varepsilon}\right\rangle} \leq P_{x}\left\langle w, X^{\varepsilon}\right\rangle .
\end{gathered}
$$

By (3.2) and 4.4.D, $\lim V_{D_{\varepsilon}}\left(u^{\prime \prime}\right)=Q_{B}\left(u^{\prime \prime}\right)=0$. Therefore $I_{\varepsilon} \rightarrow 0$. By (1.12), $P_{x}\left\langle w, X^{\varepsilon}\right\rangle=\Pi_{x} w\left(\xi_{\tau_{\varepsilon}}\right)$, where $\tau_{\varepsilon}$ is the first exit time from $D_{\varepsilon}$. By (5.2), $w=0$ on $O$, and therefore $\Pi_{x} w\left(\xi_{\tau_{\varepsilon}}\right) \rightarrow 0$. Therefore $J_{\varepsilon} \rightarrow 0$. Hence, $Q_{B}(u)=\lim u_{\varepsilon}=u^{\prime}$.

Formula (5.5) follows from 4.4.C and 3.3.C.

Lemma 5.1. Every moderate compact set $B \subset \Gamma$ for $a(\Gamma, \nu)$-solution u is $\mathcal{R}$-polar.

Proof. Put $w(x)=-\log P_{x}\{\mathcal{R} \cap B=\emptyset\}$. By Theorem 3.1, $w_{B}=Q_{B}(w)$ is the maximal element of $\mathcal{U}$ subject to the conditions $w_{B} \leq w$ and $w_{B}=0$ on $\partial E \backslash B$. Since these conditions hold for $w$, we have $w=w_{B}$. Since $u \geq w$, $u_{B}=Q_{B} u \geq w_{B}=w$ by 3.1.A. Since $u_{B}$ is a moderate solution, $w$ is also moderate and

$$
h=w+\mathcal{E}(w)
$$


is the minimal $L$-harmonic majorant of $w$. Since trace $(2 h)=2$ trace $(h)$, it follows from 3.4.B that

$$
v+\mathcal{E}(v)=2 h
$$

for some $v \geq 0$. By 4.4.B, $v \leq 2 w$, and therefore $v=0$ on $\partial E \backslash B$. But $w$ is the maximal solution with this property, and therefore $v \leq w$, which implies $2 h \leq h$ and $h=0$. Hence $w=0$ and $B$ is $\mathcal{R}$-polar.

5.3. Normal pairs. We denote by $\operatorname{Ex}(\nu)$ the set of all explosion points of a measure $\nu$. If $B \cap E x(\nu)=\emptyset$ and $B$ is compact, then $\nu(B)<\infty$. A measure $\nu$ concentrated on an open set $O$ is a Radon measure on $O$ if and only if $O \cap E x(\nu)=\emptyset$.

Note that a $(\Gamma, \nu)$-solution is at the same time a $\left(\Gamma_{0}, \nu\right)$-solution if $\Gamma_{0} \subset \Gamma$ and $\Lambda=\Gamma \backslash \Gamma_{0}$ satisfies condition (1.19). Indeed, (1.19) implies that $\{\mathcal{R} \cap \Gamma=\emptyset\}=$ $\left\{\mathcal{R} \cap \Gamma_{0}=\emptyset\right\} P_{x}$-a.s. for all $x \in E$. We call $(\Gamma, \nu)$ a normal pair if condition (1.19) holds only for $\Lambda=\emptyset$.

Lemma 5.2. Every $(\Gamma, \nu)$-solution $u$ is also a $\left(\Gamma_{0}, \nu\right)$-solution with a normal pair $\left(\Gamma_{0}, \nu\right)$.

Proof. The class $\mathcal{L}(\Gamma, \nu)$ of all sets $\Lambda$ subject to condition (1.19) is closed under union. ${ }^{9}$ Denote by $\Lambda_{0}$ the union of all $\Lambda \in \mathcal{L}(\Gamma, \nu)$, and put $\Gamma_{0}=\Gamma \backslash \Lambda_{0}$. Since $\Lambda_{0} \in \mathcal{L}(\Gamma, \nu), u$ is a $\left(\Gamma_{0}, \nu\right)$-solution. If $\Lambda \in \mathcal{L}\left(\Gamma_{0}, \nu\right)$, then $\Lambda \cup \Lambda_{0} \in \mathcal{L}(\Gamma, \nu)$, and therefore $\Lambda \subset \Lambda_{0}$. On the other hand, $\Lambda \subset \Gamma_{0}=\Gamma \backslash \Lambda_{0}$. Hence, $\Lambda=\emptyset$.

\section{4 .}

Theorem 5.2. Let $u$ be a $(\Gamma, \nu)$-solution. If $(\Gamma, \nu)$ is a normal pair, then the trace of $u$ is equal to $(\Gamma, \nu)$.

Proof. Denote the trace of $u$ by $\left(\Gamma_{0}, \nu_{0}\right)$. By the definition of the trace (see Section 3.5), $O_{0}=\partial E \backslash \Gamma_{0}$ is the maximal moderate open set for $u$. Hence, $O_{0} \supset O=\partial E \backslash \Gamma$.

For every compact set $B \subset O$, the trace of $Q_{B}(u)$ is equal to the restriction of $\nu_{0}$ to $B$. By Theorem 5.1, it is equal to the restriction of $\nu$ to $B$. Therefore $\nu=\nu_{0}$ on $O$. Since $\nu$ is concentrated on $O$, we have $\nu \leq \nu_{0}$, and therefore $\operatorname{Ex}(\nu) \subset E x\left(\nu_{0}\right) \subset \Gamma_{0} \subset \Gamma$. The set $\Lambda=\Gamma \backslash \Gamma_{0}$ is $\mathcal{R}$-polar. Indeed, every compact $B \subset \Lambda$ is moderate for $u$ (because $B \subset O_{0}$ ), and it is $\mathcal{R}$-polar by Lemma 5.1. Thus, $\Lambda$ is $\mathcal{R}$-polar, $\Lambda \cap E x(\nu)=\emptyset$ and $\Gamma \backslash \Lambda=\Gamma_{0}$ is closed. By the definition of a normal pair, this implies $\Lambda=\emptyset$.

\section{5 .}

Theorem 5.3. The trace $(\Gamma, \nu)$ of every solution $u$ is a normal pair.

First we prove two lemmas.

Lemma 5.3. If $D_{1}, D_{2}, \tilde{D}$ are open subsets of $E$ and if

$$
D_{1} \cap \tilde{D}=D_{2} \cap \tilde{D},
$$

then

$$
\{\mathcal{R} \subset \tilde{D}\} \subset\left\{X_{\tilde{D}}=0\right\} \subset\left\{X_{D_{1}}=X_{D_{2}}\right\} \quad P_{x} \text {-a.s. }
$$

\footnotetext{
${ }^{9}$ The union of an arbitrary family of $\mathcal{R}$-polar sets relatively open in $\Gamma$ is $\mathcal{R}$-polar.
} 
Proof. Let $B_{n}$ be compact sets such that $B_{n} \uparrow \tilde{D}$. By [2, Lemma 2.1], $\left\{\mathcal{R} \subset B_{n}\right\} \subset$ $\left\{X_{\tilde{D}}=0\right\}$ a.s., which implies the first part of (5.9).

The set $Q=\left\{X_{\tilde{D}}=0, X_{D_{1}} \neq X_{D_{2}}\right\}$ belongs to $\mathcal{F}_{\supset U}$, where $U=\tilde{D} \cap D_{1}=$ $\tilde{D} \cap D_{2}$. By (1.11), $P_{x}(Q)=P_{x} P_{X_{U}}(Q)$, and, by [4, II.4.6], the second part of (5.9) will be proved if we show that $P_{\eta}(Q)=0$ for every measure $\eta \in \mathcal{M}\left(U^{c}\right)$. Let $\eta^{\prime}$ be the restriction of $\eta$ to $\tilde{D}^{c}$. Note that $X_{\tilde{D}}=\eta^{\prime} P_{\eta^{\prime}}$-a.s. If $\eta^{\prime} \neq 0$, then $P_{\eta}\left\{X_{\tilde{D}}=0\right\} \leq P_{\eta^{\prime}}\left\{X_{\tilde{D}}=0\right\}=0$ and therefore $P_{\eta}(Q)=0$. If $\eta^{\prime}=0$, then $\eta$ is concentrated on the complement of $D_{1} \cup D_{2}$. Hence, $X_{D_{1}}=X_{D_{2}}=\eta P_{\eta^{-}}$a.s., and again $\mathcal{P}_{\eta}(Q)=0$.

Lemma 5.4. If $(\Gamma, \nu)$ is the trace of $u$ and if $\Gamma$ is $\mathcal{R}$-polar and $\nu$ is finite, then $u$ is moderate.

Remark. We know (see Section 3.5) that if $u$ is moderate, then $\Gamma$ is empty.

Proof. Put $B_{n}=\{x \in \partial E: d(x, \Gamma) \geq 1 / n\}$. We have $B_{n} \uparrow O=\partial E \backslash \Gamma$. By (3.5) and (3.6),

$$
\begin{aligned}
u(x) & =-\log P_{x} \exp \{-Z\}, \\
u_{n}(x)=Q_{B_{n}}(u)(x) & =-\log P_{x} \exp \left\{-Z_{B_{n}}\right\} .
\end{aligned}
$$

The trace of $u_{n}$ is the restriction of $\nu$ to $B_{n}$, and therefore

$$
u_{n}(x)+\mathcal{E}\left(u_{n}\right)(x)=\int_{B_{n}} k(x, y) \nu(d y)<\infty .
$$

Therefore, for all $x$ and $n$,

$$
u_{n}(x) \leq h(x)=\int_{O} k(x, y) \nu(d y) .
$$

By 3.3.A, there exists $\tilde{Z}$ such that $Z_{B_{n}} \uparrow \tilde{Z} P_{x}$-a.s. for all $x$. We have

$$
-\log P_{x} e^{-\tilde{Z}}=\lim u_{n}(x) \leq h(x) .
$$

To prove that $u$ is moderate, it is sufficient to show that, for every $x$,

$$
\tilde{Z}=Z \quad P_{x} \text {-a.s. }
$$

We apply Lemma 5.3 to $D_{1}=D\left(B_{n}, \varepsilon\right), D_{2}=D(\partial E, \varepsilon), \tilde{D}=D(\Gamma, \delta)$. Condition (5.8) holds if $\varepsilon<\delta-1 / n$. We conclude from (5.9) that

$$
P_{x}\left\{\mathcal{R} \subset D(\Gamma, \delta), \exp \left[-\left\langle u, X_{D\left(B_{n}, \varepsilon\right)}\right\rangle\right]\right\}=P_{x}\left\{\mathcal{R} \subset D(\Gamma, \delta), \exp \left[-\left\langle u, X_{D(\partial E, \varepsilon)}\right\rangle\right]\right\} .
$$

By passing to the limit, first as $\varepsilon \rightarrow 0$ using 3.3.C, then as $n \rightarrow \infty$ and, finally, as $\delta \rightarrow 0$, we get

$$
P_{x}\left\{\mathcal{R} \cap \Gamma=\emptyset, e^{-\tilde{Z}}\right\}=P_{x}\left\{\mathcal{R} \cap \Gamma=\emptyset, e^{-Z}\right\} .
$$

Since $\Gamma$ is $\mathcal{R}$-polar and since $P_{x}\{\tilde{Z} \leq Z\}=1$ by 3.3.A, we get (5.10).

Proof of Theorem 5.3. Let $\Lambda \in \mathcal{L}(\Gamma, \nu)$ and let $\Gamma_{0}=\Gamma \backslash \Lambda$. The theorem will be proved if we show that $v=Q_{B_{0}}(u)$ is moderate for every closed subset $B_{0}$ of $O_{0}=\partial E \backslash \Gamma_{0}$. Indeed, this implies $O_{0} \subset O$, and therefore $\Gamma_{0} \supset \Gamma, \Lambda=\emptyset$.

Let $\left(\Gamma_{1}, \nu_{1}\right)$ be the trace of $v$. By Lemma 5.4 , it is sufficient to to prove that $\Gamma_{1}$ is $\mathcal{R}$-polar and $\nu_{1}$ is finite.

By 3.1.B, $v \leq u$, and by 3.1.A, all moderate sets for $u$ are also moderate for $v$. Hence $\Gamma_{1} \subset \Gamma$. By 3.2.B, $Q_{B}\left[Q_{B_{0}}\right](u)=0$ for all $B \subset \partial E \backslash B_{0}$. Hence, $\partial E \backslash B_{0}$ is 
moderate for $v$ and it is contained in $O_{1}=\partial E \backslash \Gamma_{1}$. We conclude that $\Gamma_{1} \subset B_{0} \cap \Gamma$ which is a subset of the $\mathcal{R}$-polar set $\Lambda$.

Note that $B_{0} \subset O \cup \Lambda$ does not contain explosion points of $\nu$ and therefore $\nu\left(B_{0}\right)<\infty$. Since $Q_{B}(v)=0$ for $B \cap B_{0}=\emptyset$, the measure $\nu_{1}$ vanishes on $\partial E \backslash B_{0}$. Since $v \leq u, \nu_{1} \leq \nu$ on $O \subset O_{1}$. We have

$$
\nu_{1}\left(O_{1}\right)=\nu_{1}\left(O_{1} \cap B_{0}\right)=\nu_{1}\left[\left(O_{1} \backslash O\right) \cap B_{0}\right]+\nu_{1}\left(O \cap B_{0}\right) \leq \nu\left(O \cap B_{0}\right)<\infty
$$

because $\Gamma \cap B_{0}$ is polar, and therefore $\nu_{1}\left(\Gamma \cap B_{0}\right)=0$ by Theorem 1.1.

\section{MaXimal PROPERTy of $(\Gamma, \nu)$-SOlutions}

6.1. In this section we consider an arbitrary solution $u$ with the trace $(\Gamma, \nu)$. By 3.5.A, $\nu$ does not charge $\mathcal{R}$-polar sets, and, by Theorems 4.3 and 4.2 , it is the spectral measure of a CLA functional $A^{\nu}$. Our objective is to prove:

Theorem 6.1. We have

$$
u(x) \leq-\log P_{x}\left\{\mathcal{R} \cap \Gamma=\emptyset, e^{-A^{\nu}}\right\} .
$$

This follows easily from a kind of a mean value theorem which is of independent interest:

Theorem 6.2. Let $u$ be a solution with the trace $(\nu, \Gamma)$. For every regular open subset $D$ of $E$ such that $\partial D \cap \Gamma=\emptyset$, there exists a random variable $Z^{D}$ with the properties

$$
\begin{gathered}
Z^{D} \leq A^{\nu} ; \\
u(x)=-\log P_{x} e^{-Z^{D}-\left\langle u, X_{D}\right\rangle} \quad \text { in } D .
\end{gathered}
$$

To prove Theorem 6.1 , we apply Theorem 6.2 to domains $D_{n}=D(\Gamma, 1 / n)$. It follows from (6.2) and (6.3) that

$$
u(x) \leq-\log P_{x} e^{-A^{\nu}} e^{-\left\langle u, X_{D_{n}}\right\rangle}
$$

for every $n$. Since $\left\langle u, X_{D_{n}}\right\rangle \rightarrow 0$ on the set $\{\mathcal{R} \cap \Gamma=\emptyset\}$, we have liminf $e^{-\left\langle u, X_{D_{n}}\right\rangle} \geq$ $1_{\mathcal{R} \cap \Gamma=\emptyset}$, and Fatou's lemma implies that

$$
\liminf P_{x} e^{-A^{\nu}} e^{-\left\langle u, X_{D_{\varepsilon}}\right\rangle} \geq P_{x}\left\{\mathcal{R} \cap \Gamma=\emptyset, e^{-A^{\nu}}\right\} .
$$

Equations (6.4) and (6.5) imply (6.1).

\section{2 .}

Proof of Theorem 6.2. $1^{\circ}$. Put $B=\partial D \cap \partial E, D_{n}=D(B, 1 / n)$ and $\tilde{D}_{n}=D \cap D_{n}$. For every set $A$, put $u_{A}=u 1_{A}$.

By the mean value property 2.1.D,

$$
u(x)=-\log P_{x} e^{-\left\langle u, X_{\tilde{D}_{n}}\right\rangle} \quad \text { in } \tilde{D}_{n} .
$$

For every $x \in \tilde{D}_{n}$ the measure $X_{\tilde{D}_{n}}$ is concentrated, $P_{x^{-}}$a.s., on $C \cup D$, where $C=\partial D \cap E$, and therefore

$$
\left\langle u, X_{\tilde{D}_{n}}\right\rangle=\left\langle u_{C}, X_{\tilde{D}_{n}}\right\rangle+\left\langle u_{D}, X_{\tilde{D}_{n}}\right\rangle \quad P_{x^{-}} \text {a.s. }
$$

Theorem 6.2 will follow from (6.6) if we prove that, for every $x \in D$,

$$
\lim \left\langle u_{C}, X_{\tilde{D}_{n}}\right\rangle=\left\langle u, X_{D}\right\rangle \quad P_{x^{-} \text {a.s. }}
$$


and

$$
\left\langle u_{D}, X_{\tilde{D}_{n}}\right\rangle \rightarrow Z^{D} \quad P_{x} \text {-a.s. }
$$

where $Z^{D}$ satisfies (6.2).

$2^{\circ}$. Note that $\left\langle u_{C}, X_{\tilde{D}_{n}}\right\rangle=\left\langle u, \tilde{X}_{n}\right\rangle$ and that, for all $x \in D,\left\langle u, X_{D}\right\rangle=\langle u, \tilde{X}\rangle$ $P_{x^{-}}$a.s., where $\tilde{X}_{n}$ and $\tilde{X}$ are the restrictions of $X_{\tilde{D}_{n}}$ and $X_{D}$ to $C$. Therefore we get (6.7) if we prove that $\tilde{X}_{n} \uparrow \tilde{X} P_{x}$-a.s.

By 2.1.E, $\tilde{X}_{n} \leq \tilde{X}_{n+1} \leq \tilde{X}$ a.s., and therefore

$$
\tilde{X}_{n} \uparrow X^{*} \leq \tilde{X} \quad \text { a.s. }
$$

By (1.10) and (1.12),

$$
\begin{aligned}
P_{x}\left\langle 1, \tilde{X}_{n}\right\rangle & =\Pi_{x}\left\{\xi_{\tilde{\tau}_{n}} \in C\right\}, \\
P_{x}\langle 1, \tilde{X}\rangle & =\Pi_{x}\left\{\xi_{\tau} \in C\right\},
\end{aligned}
$$

where $\tilde{\tau}_{n}, \tau$ are the first exit moments from $\tilde{D}_{n}, D$. If $x \in D$, then $\left\{\xi_{\tilde{\tau}_{n}} \in C\right\} \uparrow$ $\left\{\xi_{\tau} \in C\right\} \Pi_{x}$-a.s., and (6.9) implies that $X^{*}=\tilde{X} P_{x}$-a.s.

$3^{\circ}$. Denote by $\bar{X}_{n}$ the restriction of $X_{\tilde{D}_{n}}$ to $D$. Note that $\bar{X}_{n+1}=0 P_{\tilde{X}_{n}}$-a.s. By (1.11) and (1.7),

$$
\begin{aligned}
P_{x}\left\{e^{-\left\langle u_{D}, X_{\tilde{D}_{n+1}}\right\rangle} \mid \mathcal{F}_{\subset \tilde{D}_{n}}\right\} & =P_{\tilde{X}_{\tilde{D}_{n}}} e^{-\left\langle u_{D}, X_{\tilde{D}_{n+1}}\right\rangle}=P_{\bar{X}_{n}} e^{-\left\langle u_{D}, X_{\tilde{D}_{n+1}}\right\rangle} \\
& \geq P_{\bar{X}_{n}} e^{-\left\langle u, X_{\tilde{D}_{n+1}}\right\rangle}=e^{-\left\langle u, \bar{X}_{n}\right\rangle}=e^{-\left\langle u_{D}, X_{\tilde{D}_{n}}\right\rangle} .
\end{aligned}
$$

Therefore $e^{-\left\langle u_{D}, X_{\tilde{D}_{n}}\right\rangle}$ is a bounded submartingale relative to $P_{x}$ (cf. the proof of 3.3.C) and there exists $Z^{D}$ subject to (6.8).

To get (6.2), we recall that, by Section 3.5, the restriction $\nu_{B}$ of $\nu$ to $B$ is the trace of $Q_{B}(u)$. By 4.4.C, the corresponding functional $A^{\nu_{B}}$ satisfies the condition

$$
A^{\nu_{B}}=\lim \left\langle u, X_{D_{n}}\right\rangle \quad \text { a.s. }
$$

Proposition 2.1.E implies that

$$
\left\langle u_{D}, X_{\tilde{D}_{n}}\right\rangle \leq\left\langle u_{D}, X_{D_{n}}\right\rangle \leq\left\langle u, X_{D_{n}}\right\rangle
$$

Hence

$$
Z^{D}=\lim \left\langle u_{D}, X_{\tilde{D}_{n}}\right\rangle \leq A^{\nu_{B}} \leq A^{\nu}
$$

\section{REFERENCES}

1. E. B. Dynkin, Markov Processes, Springer-Verlag, Berlin, 1965. MR 33:1887

2. _ A probabilistic approach to one class of nonlinear differential equations, Probab. Th. Rel. Fields 89 (1991), 89-115. MR 92d:35090

3. (1992), 942-962. MR 93d:60124

4. - Superprocesses and partial differential equations, Ann. Probab. 21 (1993), 11851262. MR 94j:60156

5. __ An Introduction to Branching Measure-Valued Processes, American Mathematical Society, Providence, Rhode Island, 1994. MR 96f:60145

6. E. B. Dynkin and S. E. Kuznetsov, Superdiffusions and removable singularities for quasilinear partial differential equations, Comm. Pure \& Appl. Math. 49 (1996), 125-176. MR 97m:60144

7. , Solutions of $L u=u^{\alpha}$ dominated by L-harmonic functions, Journal d'Analyse Mathématique 68 (1996), 15-37. MR 97f:35048 
8. Linear additive functionals of superdiffusions and related nonlinear P.D.E., Trans. Amer. Math. Soc. 348 (1996), 1959-1987. MR 97d:60135

9. - Nonlinear parabolic P.D.E. and additive functionals of superdiffusions, Ann. Probab. 25 (1997), 662-701. CMP 97:08

10. _ Natural linear additive functionals of superprocesses, Ann. Probab. 25 (1997), 640661. CMP 97:08

11. Solutions of nonlinear differential equations on a Riemannian manifold and their trace on the Martin boundary, Trans. Amer. Math. Soc., 350 (1998), 4521-4552. CMP 97:11

12. A. Gmira and L. Véron, Boundary singularities of solutions of some nonlinear elliptic equations, Duke Math.J. 64 (1991), 271-324. MR 93a:35053

13. J.F. Le Gall, Solutions positives de $\Delta u=u^{2}$ dans le disque unité, C.R. Acad. Sci. Paris, Série I 317 (1993), 873-878. MR 94h:35059

14. The Brownian snake and solutions of $\Delta=u^{2}$ in a domain, Probab. Theory Relat. Fields 102 (1995), 393-402. MR 96c:60098

15. - A probabilistic Poisson representation for positive solutions of $\Delta u=u^{2}$ in a planar domain, Comm. Pure Appl. Math. 50 (1997), 69-103. MR 98c:60144

16. - A probabilistic approach to the trace at the boundary for solutions of a semilinear parabolic partial differential equation, J. Appl. Math. Stochastic Anal. 9 (1996), 399-414. MR 97m:35125

17. M. Marcus and L. Véron, Trace au bord des solutions positives d'équations elliptiques non linéaires, C.R. Acad. Sci Paris Sér. I Math. 321 (1995), 179-184. MR 96f:35045

18. __ Trace au bord des solutions positives d'équations elliptiques et paraboliques non linéaires. Résultats d'existence et d'unicité, C.R. Acad. Sci Paris Sér. I Math. 323 (1996), 603-608. MR 97f:35012

19. V. G. Maz'ya, Beurling's theorem on a minimum principle for positive harmonic functions, [First published (in Russian) in Zap. Nauchn. Sem. Leningrad. Otdel. Mat. Inst. Steklov. 30 (1972), 76-90], J.Soviet Math. 4 (1975) 367-379. MR 48:8821

Department of Mathematics, Cornell University, Ithaca, New York 14853-7901

E-mail address: ebd1@cornell.edu

Department of Mathematics, University of Colorado at Boulder, Boulder, ColORADO 80309-0395

E-mail address: sk47@cornell.edu 